\title{
Impulsive differential equations with nonlocal conditionsin general Banach spaces
}

\author{
Lanping Zhu*, Qixiang Dong and Gang Li
}

* Correspondence: Ipzmath@yahoo.
com.cn
School of Mathematics, Yangzhou
University, Yangzhou 225002, China

\section{Abstract}

This article is concerned with impulsive semilinear differential equations with nonlocal initial conditions in Banach spaces. The approach used is fixed point theorem combined with the technique of operator transformation. Existence results are obtained when the nonlocal item is Lipschitz continuous. An example is also given to illustrate the obtained theorem.

AMS classification: 34G10; 47D06.

Keywords: impulsive differential equations, measure of noncompactness, fixed point theorem, mild solutions

\section{Introduction}

In this article, we deal with the existence of mild solutions for the following impulsive semilinear nonlocal problem

$$
\left\{\begin{array}{l}
u^{\prime}(t)=A u(t)+f(t, u(t)), \quad t \in[0, T], \quad t \neq t_{i}, \\
u(0)=g(u), \\
\Delta u\left(t_{i}\right)=I_{i}\left(u\left(t_{i}\right)\right), \quad i=1,2, \ldots, p, \quad 0<t_{1}<t_{2}<\cdots<t_{p}<T,
\end{array}\right.
$$

where $A: D(A) \subseteq X \rightarrow X$ is the infinitesimal generator of strongly continuous semigroup $S(t)$ for $t>0$ in a real Banach space $X, \Delta u\left(t_{i}\right)=u\left(t_{i}^{+}\right)-u\left(t_{i}^{-}\right)$constitutes an impulsive condition. $f$ and $g$ are $X$-valued functions to be given later.

In recent years, the theory of impulsive differential inclusions has become an important object of investigation because of its wide applicability in biology, medicine, mechanics, control and in more and more fields. The impulsive conditions are the appropriate model for describing some phenomena. For example, at certain moments, the system changes their state rapidly, which cannot be modeled by traditional initial value problems. For more detailed bibliography and exposition on this subject, we refer to [1-6].

Here we first recall the study of nonlocal semilinear initial value problems. It was first considered by Byszewski. Because it has better effect in the applications than the classical initial condition, more and more authors have studied the following type of semilinear differential equation under various conditions on $S(t), f$, and $g$,

$$
\left\{\begin{array}{l}
u^{\prime}(t)=A u(t)+f(t, u(t)), t \in[0, T] \\
u(0)=g(u)
\end{array}\right.
$$


For instance, Byszewski and Lakshmikantham [7] proved the existence and uniqueness of mild solutions for nonlocal semilinear differential equations when $f$ and $g$ satisfy Lipschitz type conditions. In [8], Ntouyas and Tsamatos studied the case with compactness conditions. Byszewski and Akca [9] established the existence of solution to functional-differential equation when the semigroup is compact, and $g$ is convex and compact on a given ball. Subsequently, Benchohra and Ntouyas [10] discussed second order differential equation under compact conditions. Recently, Dong and Li [11] study the semilinear differential inclusion when $g$ is compact. By making full use of the measure of noncompactness, Obukhovski and Zecca [12] discuss the controllability for semilinear differential inclusions with a noncompact semigroup, Xue [13-15] established new existence theorems for semilinear and nonlinear nonlocal problem, respectively.

Next, we focus on the study of impulsive problems. Liu [5] discuss the classic initial problem when $f$ is Lipschitz continuous with respect to its second variable and the impulsive functions $I_{i}$ are Lipschitz continuous. Cardinali and Rubbioni [3] study the multivalued impulsive semilinear differential equation by means of the Hausdorff measure of noncompactness. Liang et al. [16] investigate the nonlocal impulsive problems under the assumptions of $g$ is compact, Lipschitz, and $g$ is not compact and not Lipschitz, respectively.

The goal of this article is to make use of the Hausdorff measure of noncompactness and the fixed point theory to deal with the impulsive semilinear differential equation (1.1). We obtain the existence of mild solution of the nonlocal problem (1.1) when $g$ is Lipschitz continuous. In particular, in our proof, we do not need the Lipschitz continuity of $f$. Thus the compactness of $S(t)$ or $f$ and the Lipschitz continuity of $f$ are the special case of our results.

This article is organized as follows. In Section 2, we recall some facts about the measure of noncompactness, fixed point theorem and semilinear differential equations. In Section 3, we give the existence result of the problem (1.1) when $g$ is Lipschitz continuous. In Section 4, an example is given to illustrate our abstract results.

\section{Preliminaries}

Let $E$ be a real Banach space, we introduce the Hausdorff measure of noncompactness $\alpha$ defined on each bounded subset $\Omega$ of $E$ by

$$
\alpha(\Omega)=\inf \left\{r>0 \text {; there are finite points } x_{1}, x_{2}, \ldots, x_{n} \in E \text { with } \Omega \subset \cup_{i=1}^{n} B\left(x_{i}, r\right)\right\} .
$$

Now, we recall some basic properties of the Hausdorff measure of noncompactness.

Lemma 2.1 [17]. For all bounded subsets $\Omega, \Omega_{1}, \Omega_{2}$ of $E$, the following properties are satisfied:

(1) $\Omega$ is precompact if and only if $\alpha(\Omega)=0$;

(2) $\alpha(\Omega)=\alpha(\bar{\Omega})=\alpha(\operatorname{conv} \Omega)$, where $\bar{\Omega}$ and $\operatorname{conv} \Omega$ mean the closure and convex hull of $\Omega$, respectively;

(3) $\alpha(\Omega 1) \leq \alpha\left(\Omega_{2}\right)$ when $\Omega 1 \subset \Omega_{2}$;

(4) $\alpha\left(\Omega_{1} \cup \Omega_{2}\right) \leq \max \left\{\alpha\left(\Omega_{1}\right), \alpha\left(\Omega_{2}\right)\right\}$;

(5) $\alpha(\lambda \Omega)=|\lambda| \alpha(\Omega)$, for any $\lambda \in R$;

(6) $\alpha\left(\Omega_{1}+\Omega_{2}\right) \leq \alpha\left(\Omega_{1}\right)+\alpha\left(\Omega_{2}\right)$, where $\Omega_{1}+\Omega_{2}=\left\{x+y ; x \in \Omega_{1}, y \in \Omega_{2}\right\}$; 
(7) if $\left\{W_{n}\right\}_{n=1}^{+\infty}$ is a decreasing sequence of nonempty bounded closed subsets of $E$ and $\lim _{n \rightarrow \infty} \alpha\left(W_{n}\right)=0$, then $\cap_{n=1}^{+\infty} W_{n}$ is nonempty and compact in $E$.

The map $Q: D \subset E \rightarrow E$ is said to be an $\alpha$-contraction, if there exists a positive constant $k<1$ such that $\alpha(Q B)<k \alpha(B)$ for every bounded closed subset $B \subset D$ (see [18]).

Lemma 2.2 ([17]: Darbo-Sadovskii). If $D \subset E$ is bounded closed and convex, the continuous map $Q: D \rightarrow D$ is an $\alpha$-contraction, then the map $Q$ has at least one fixed point in $D$.

Throughout this article, let $(X,\|\|$.$) be a real Banach space. We denote by C([0, T] ; X)$ the Banach space of all continuous functions from $[0, T]$ to $X$ with the norm $\|u\|=\sup$ $\{\|u(t)\|, t \in[0, T]\}$ and by $L^{1}([0, T] ; X)$ the Banach space of all $X$-valued Bochner integrable functions defined on $[0, T]$ with the norm $\|u\|_{1}=\int_{0}^{T}\|u(t)\| d t$. Let $P C([0, T] ; X)=$ $\left\{u:[0, T] \rightarrow X: u(t)\right.$ be continuous at $t \neq t_{i}$ and left continuous at $t=t_{i}$ and the right limit $u\left(t_{i}^{+}\right)$exists for $\left.i=1,2, \ldots, p\right\}$. It is easy to check that $P C([0, T] ; X)$ is a Banach space with the norm $\|u\|_{P C}=\sup \{\|u(t)\|, t \in[0, T]\}$ and $C([0, T] ; X) \subseteq P C([0, T] ; X) \subseteq L^{1}([0, T] ; X)$. Moreover, we denote $\beta$ by the Hausdorff measure of noncompactness of $X$, denote $\beta_{c}$ by the Hausdorff measure of noncompactness of $C([0, T] ; X)$ and denote $\beta_{p c}$ by the Hausdorff measure of noncompactness of $P C([0, T] ; X)$.

$C_{0}$-semigroup $S(t)$ is said to be equicontinuous if $\{S(t) x: x \in B\}$ is equicontinuous for $t>0$ for all bounded set $B \subset E$. Consequently, the following lemma is easily verified.

Lemma 2.3 If the semigroup $S(t)$ is equicontinuous and $w \in L^{1}\left([0, T] ; R^{+}\right)$, then the $\operatorname{set}\left\{\int_{0}^{t} S(t-s) u(s) d s,\|u(s)\| \leq w(s),\|u(s)\| \leq w(s)\right.$ for a.e. $\left.s \in[0, T]\right\}$ is equicontinuous for $t \in[0, T]$.

Lemma 2.4 If $W \subseteq P C([0, T] ; X)$ is bounded, then we have

$$
\sup _{t \in[0, T]} \beta(W(t)) \leq \beta_{p c}(W),
$$

where $W(t)=\{u(t) ; u \in W\} \subset X$.

Proof. For arbitrary $\varepsilon>0$, there exists $W_{i} \subseteq P C([0, T] ; X), i=1,2, \ldots, n$, such that $W \subseteq \cup_{i=1}^{n} W_{i}$ and

$$
\operatorname{diam}\left(W_{i}\right) \leq 2 \beta_{p c}(W)+\varepsilon, \quad i=1,2, \ldots, n .
$$

Hence, for every $t \in[0, T], W(t) \subseteq \cup_{i=1}^{n} W_{i}(t)$, and $\operatorname{diam}\left(W_{i}(t)\right) \leq \operatorname{diam}\left(W_{i}\right), i=1$, $2, \ldots, n$, that is

$$
2 \beta(W(t)) \leq \max _{1 \leq i \leq n} \operatorname{diam}\left(W_{i}(t)\right) \leq \max _{1 \leq i \leq n} \operatorname{diam}\left(W_{i}\right) \leq 2 \beta_{p c}(W)+\varepsilon,
$$

and therefore,

$$
\sup _{t \in[0, T]} \beta(W(t)) \leq \beta_{p c}(W) .
$$

To discuss the problem (1.1), we also need the following lemma.

Lemma 2.5 [4]. If $W \subseteq C([0, T] ; X)$ is bounded, then for all $t \in[0, T]$,

$$
\beta(W(t)) \leq \beta_{c}(W),
$$

where $W(t)=\{u(t) ; u \in W\} \subset X$. Furthermore, if $W$ is equicontinuous on $[0, T]$, then $\beta$ $(W(t))$ is continuous on $[0, T]$ and 


$$
\beta_{c}(W)=\sup \{\beta(W(t)): t \in[0, T]\} .
$$

We will also use the sequential measure of noncompactness $\beta_{0}$ generated by $\beta$, that is, for any bounded subset $\Omega \subset X$, we define

$$
\beta_{0}(\Omega)=\sup \left\{\beta\left(\left\{x_{n}: n \geq 1\right\}\right):\left\{x_{n}\right\}_{n=1}^{+\infty} \text { is a sequence in } \Omega\right\} .
$$

It follows that

$$
\beta_{0}(\Omega) \leq \beta(\Omega) \leq 2 \beta_{0}(\Omega) .
$$

In addition, when $X$ is separable, we have $\beta_{0}(\Omega)=\beta(\Omega)$.

For the above related results on the sequential measure of noncompactness, we refer to [17].

Definition 2.1 A function $u \in C([0, T] ; X)$ is said to be a mild solution of the nonlocal problem (1.2), if

$$
u(t)=S(t) g(u)+\int_{0}^{t} S(t-s) f(s, u(s)) d s
$$

for all $t \in[0, T]$.

Let $\Gamma f$ be the only mild solution of the following semilinear system

$$
\begin{aligned}
& u^{\prime}(t)=A u(t)+f(t), \quad \text { a.e. } t \in[0, T], \\
& u(0)=u_{0} .
\end{aligned}
$$

Now, we give the following result about $\beta$-estimation of mild solutions (see [19]), similarly, see also $[20,21]$.

Lemma 2.6 Let $\left\{f_{k}\right\}_{k=1}^{+\infty}$ be a sequence of functions in $L^{1}([0, T] ; X)$. Assume that there exists $\phi \in L^{1}([0, T] ; R+)$ satisfying

$$
\left\|f_{k}(t)\right\| \leq \varphi(t) \text { a.e on }[0, T] \text { for all } k \geq 1 .
$$

Then for all $t \in[0, T]$, we have

$$
\beta\left(\left\{\left(\Gamma f_{k}\right)(t): k \geq 1\right\}\right) \leq 2 M \int_{0}^{t} \beta\left(\left\{f_{k}(s): k \geq 1\right\}\right) d s .
$$

Definition 2.2 A function $u \in P C([0, T] ; X)$ is said to be a mild solution of the nonlocal problem (1.1), if it satisfies

$$
u(t)=S(t) g(u)+\int_{0}^{t} S(t-s) f(s, u(s)) d s+\sum_{0<t_{i}<t} S\left(t-t_{i}\right) I_{i}\left(u\left(t_{i}\right)\right), \quad 0 \leq t \leq T .
$$

Since $\{S(t): t \in[0, T]\}$ is a strongly continuous semigroup of bounded linear operators, we may assume $\|S(t)\| \leq M$ for all $t \in[0, T]$. In addition, let $r$ be a finite positive constant, and set $B_{r}:=\{x \in X:\|x\| \leq r\}$ and $W_{r}:=\left\{u \in P C([0, T] ; X): u(t) \in B_{r}, \forall t \in\right.$ $[0, T]\}$. 


\section{Main results}

In this section, by using the method and technique of operator transformation, Hausdorff measure of noncompactness and fixed point, we give the existence result for the nonlocal problem (1.1). First, we give the following hypotheses:

$\left(H_{A}\right)$ The $C_{0}$ semigroup $S(t)$ generated by $A$ is equicontinuous;

$\left(H_{f}\right) f:[0, T] \times X \rightarrow X$ satisfies the following conditions:

(1) $f(\cdot, x):[0, T] \rightarrow X$ is measurable for all $x \in X$,

(2) $f(t, \cdot): X \rightarrow X$ is continuous for a.e. $t \in[0, T]$,

(3) there exists $l(t) \in L^{1}\left(0, T ; R^{+}\right)$such that

$$
\beta(f(t, D) \leq l(t) \beta(D),
$$

for a.e. $t \in[0, T]$ and every bounded subset $D \subset X$;

( $\left.H_{I}\right) I_{i}: X \rightarrow X$ is Lipschitz continuous with Lipschitz constant $k_{i}$, for $i=1,2, \ldots, p$;

$\left(H_{g}\right)$ There exists a constant $k \in\left(0,1 / M-\sum_{i=1}^{p} k_{i}\right)$ such that

$$
\|g(u)-g(v)\| \leq k\|u-v\|, \text { for } u, v \in P C([0, T] ; X) ;
$$

$$
\left(H_{r}\right) M\left(\|g(0)\|+\sum_{i=1}^{p}\left\|I_{i}(0)\right\|+T \cdot \sup _{t \in[0, T], u \in W_{r}}\|f(t, u(t))\|\right) \leq\left(1-M\left(k+\sum_{i=1}^{p} k_{i}\right)\right) r .
$$

Theorem 3.1 Assume that the conditions $\left(H_{A}\right),\left(H_{f}\right)(1)-(3),\left(H_{I}\right),\left(H_{g}\right)$, and $\left(H_{r}\right)$ are satisfied. Then the nonlocal problem (1.1) has at least one mild solution on $[0, T]$ provided that $M\left(4 l_{1}+k+\sum_{i=1}^{p} k_{i}\right)<1$, where $l_{1}=\int_{0}^{T} l(s) d s$.

Define the operator $R: P C([0, T] ; X) \rightarrow C([0, T] ; X)$ by

$$
R u(t)=\int_{0}^{t} S(t-s) f(s, u(s)) d s, \quad 0 \leq t \leq T .
$$

Using the dominated convergence theorem, it is easy to check that $R$ is continuous on $P C([0, T] ; X)$ by the continuity of $f$ with respect to the second argument. Furthermore, by the assumption $\left(H_{r}\right)$ and Lemma 2.3, we know $R\left(W_{r}\right)$ is bounded and equicontinuous on $[0, T]$.

To prove the above theorem, we first give the following lemma.

Lemma 3.1 If the condition $\left(H_{r}\right)$ holds, then for arbitrary bounded set $W \subset W_{r}$, we have

$$
\beta(R W(t)) \leq 4 M \int_{0}^{t} \beta(f(s, W(s)) d s, \quad t \in[0, T] .
$$

Proof. For any $t \in[0, T]$, due to the inequality (2.1), we obtain that for arbitrary given $\varepsilon>0$, there exists a sequence $\left\{v_{k}\right\}_{k=1}^{+\infty} \subset W$ such that

$$
\beta(R W(t)) \leq 2 \beta\left(\left\{R v_{k}(t): k \geq 1\right\}\right)+\varepsilon .
$$


Since

$$
\left(\Gamma f\left(\cdot, v_{k}(\cdot)\right)\right)(t)=S(t) u_{0}+\int_{0}^{t} S(t-s) f\left(s, v_{k}(s)\right) d s \text { for all } t \in[0, T]
$$

we obtain

$$
\begin{aligned}
\left.\beta\left(\left\{\Gamma f\left(\cdot, v_{k}(\cdot)\right)\right)(t): k \geq 1\right\}\right) & =\beta\left(\left\{\left(S(t) u_{0}+\int_{0}^{t} S(t-s) f\left(s, v_{k}(s)\right) d s\right): k \geq 1\right\}\right) \\
& =\beta\left(\left\{R v_{k}(t): k \geq 1\right\}\right) .
\end{aligned}
$$

On the other hand, it follows from Lemma 2.6 that

$$
\beta\left(\left\{\left(\Gamma f\left(\cdot, v_{k}(\cdot)\right)\right)(t): k \geq 1\right\}\right) \leq 2 M \int_{0}^{t} \beta\left(\left\{f\left(s, v_{k}(s)\right): k \geq 1\right\}\right) d s .
$$

Hence, we have

$$
\beta\left(\left\{R v_{k}(t): k \geq 1\right\}\right) \leq 2 M \int_{0}^{t} \beta\left(\left\{f\left(s, v_{k}(s)\right): k \geq 1\right\}\right) d s .
$$

By applying (3.1), we can derive that

$$
\beta(R W(t)) \leq 4 M \int_{0}^{t} \beta\left(\left\{f\left(s, v_{k}(s)\right)\right) d s: k \geq 1\right\} d s+\varepsilon .
$$

Since

$$
\beta\left(\left\{f\left(s, v_{k}(s)\right)\right): k \geq 1\right\} \leq \beta(f(s, W(s))),
$$

we infer that

$$
\beta(R W(t)) \leq 4 M \int_{0}^{t} \beta(f(s, W(s)) d s+\varepsilon .
$$

Since the above inequality holds for arbitrary $\varepsilon>0$, it follows that

$$
\beta(R W(t)) \leq 4 M \int_{0}^{t} \beta(f(s, W(s)) d s .
$$

This completes the proof.

Proof of Theorem 3.1 Define the operator $Q: P C([0, T] ; X) \rightarrow P C([0, T] ; X)$ by

$$
(Q u)(t)=u(t)-S(t) g(u)-\sum_{0<t_{i}<t} S\left(t-t_{i}\right) I_{i}\left(u\left(t_{i}\right)\right), \quad 0 \leq t \leq T .
$$

Obviously, the fixed point of $Q^{-1} R$ is the mild solution of the nonlocal impulsive problem (1.1). Subsequently, we will prove that $Q^{-1} R$ has a fixed point by Lemma 2.2. 
At first, we prove that $Q$ is Lipschitz continuous with constant $1+M\left(k+\sum_{i=1}^{p} k_{i}\right)$. In fact, by using the conditions $\left(H_{g}\right)$ and $\left(H_{I}\right)$, for $u_{1}, u_{2} \in P C([0, T] ; X)$, we have

$$
\begin{aligned}
\left\|\left(Q u_{1}\right)(t)-\left(Q u_{2}\right)(t)\right\| \leq & \left\|u_{1}(t)-u_{2}(t)\right\|+\left\|S(t) g\left(u_{1}\right)-S(t) g\left(u_{2}\right)\right\| \\
& +\sum_{i=1}^{p}\left\|S\left(t-t_{i}\right) I_{i}\left(u_{1}\left(t_{i}\right)\right)-S\left(t-t_{i}\right) I_{i}\left(u_{2}\left(t_{i}\right)\right)\right\| \\
\leq & \left\|u_{1}-u_{2}\right\|_{P C}+M k\left\|u_{1}-u_{2}\right\|_{P C}+M \sum_{i=1}^{p} k_{i}\left\|u_{1}-u_{2}\right\|_{P C} \\
= & \left(1+M\left(k+\sum_{i=1}^{p} k_{i}\right)\right)\left\|u_{1}-u_{2}\right\|_{P C} .
\end{aligned}
$$

Secondly, we show that $Q$ is bijective. For this purpose, for any fixed $v \in P C([0, T]$; $X$ ), we consider the following equation:

$$
(Q u)(t)=u(t)-S(t) g(u)-\sum_{0<t_{i}<t} S\left(t-t_{i}\right) I_{i}\left(u\left(t_{i}\right)\right)=v(t), 0 \leq t \leq T .
$$

Now define the operator $L: P C([0, T] ; X) \rightarrow P C([0, T] ; X)$ by

$$
(L u)(t)=S(t) g(u)+\sum_{0<t_{i}<t} S\left(t-t_{i}\right) I_{i}\left(u\left(t_{i}\right)\right)+v(t), 0 \leq t \leq T .
$$

It is easy to see that the existence and uniqueness of the fixed point of $L$ for any $v \in$ $P C([0, T] ; X)$ implies that $Q$ is bijective. In the following, we will prove that $L$ has a unique fixed point in $P C([0, T] ; X)$. Indeed, for $u_{1}, u_{2} \in P C([0, T] ; X)$,

$$
\begin{aligned}
\left\|\left(L u_{1}\right)(t)-\left(L u_{2}\right)(t)\right\| \leq & \left\|S(t) g\left(u_{1}\right)-S(t) g\left(u_{2}\right)\right\| \\
& +\sum_{i=1}^{p}\left\|S\left(t-t_{i}\right) I_{i}\left(u_{1}\left(t_{i}\right)\right)-S\left(t-t_{i}\right) I_{i}\left(u_{2}\left(t_{i}\right)\right)\right\| \\
\leq & M k\left\|u_{1}-u_{2}\right\|_{P C}+M \sum_{i=1}^{p} k_{i}\left\|u_{1}-u_{2}\right\|_{P C} \\
\leq & M\left(k+\sum_{i=1}^{p} k_{i}\right)\left\|u_{1}-u_{2}\right\|_{P C} .
\end{aligned}
$$

From the condition $\left(H_{g}\right)$, we find that $M\left(k+\sum_{i=1}^{p} k_{i}\right)<1$, that is, $L$ is a contraction operator on $P C([0, T] ; X)$. According to Banach's fixed point theorem, $L$ has a unique fixed point. Thus, $Q$ is bijective.

Third, we prove that $Q^{-1}$ is Lipschitz continuous with constant $1 /\left(1-M\left(k+\sum_{i=1}^{p} k_{i}\right)\right)$. In fact, for $v_{1}, v_{2} \in P C([0, T] ; X)$,

$$
\begin{aligned}
\left\|\left(Q^{-1} v_{1}\right)(t)-\left(Q^{-1} v_{2}\right)(t)\right\| \leq & \left\|v_{1}(t)-v_{2}(t)\right\|+\left\|S(t) g\left(Q^{-1}\left(v_{1}\right)\right)-S(t) g\left(Q^{-1}\left(v_{2}\right)\right)\right\| \\
& +\sum_{i=1}^{p}\left\|S\left(t-t_{i}\right) I_{i}\left(Q^{-1}\left(v_{1}\right)\left(t_{i}\right)\right)-S\left(t-t_{i}\right) I_{i}\left(Q^{-1}\left(v_{2}\right)\left(t_{i}\right)\right)\right\| \\
\leq & \left\|v_{1}-v_{2}\right\|_{P C}+M k\left\|Q^{-1}\left(v_{1}\right)-Q^{-1}\left(v_{2}\right)\right\|_{P C} \\
& +M \sum_{i=1}^{p} k_{i}\left\|Q^{-1}\left(v_{1}\right)-Q^{-1}\left(v_{2}\right)\right\|_{P C} \\
\leq & \left.\left\|v_{1}-v_{2}\right\|_{P C}+M\left(k+\sum_{i=1}^{p} k_{i}\right)\right)\left\|Q^{-1}\left(v_{1}\right)-Q^{-1}\left(v_{2}\right)\right\|_{P C}
\end{aligned}
$$


Next, we claim that $\left(Q^{-1} R\right) W_{r} \subseteq W_{r}$. Actually, for any $u \in W_{r} \subseteq P C([0, T] ; X)$, let $v=$ $\left(Q^{-1} R\right) u$, from the hypotheses $\left(H_{I}\right)$ and $\left(H_{g}\right)$, we have

$$
\begin{aligned}
\|v(t)\| \leq & \left.\|S(t) g(v)\|+\sum_{i=1}^{p} \| S\left(t-t_{i}\right) I_{i}(v)\left(t_{i}\right)\right) \| \\
& +\int_{0}^{t}\|S(t-s)\| \sup _{t \in[0, T], u \in W_{r}} \| f(s, u(s) \| d s \\
\leq & M\left(\left(k+\sum_{i=1}^{p} k_{i}\right)\|v\|_{P C}+\|g(0)\|+\sum_{i=1}^{p}\left\|I_{i}(0)\right\|\right. \\
& \left.+T \cdot \sup _{t \in[0, T], u \in W_{r}}\|f(t, u(t))\|\right) .
\end{aligned}
$$

By the condition $\left(H_{r}\right)$, we infer that $\|v\|_{P C} \leq r$. Thus, $\left(Q^{-1} R\right) W_{r} \subseteq W_{r}$.

At last, we prove that $Q^{-1} R$ is a $\beta_{p c}$-contraction. As $Q^{-1}$ is Lipschitz continuous and $R$ is continuous on $P C([0, T] ; X)$, we have $Q^{-1} R$ is continuous on $P C([0, T] ; X)$. Actually, since $R\left(W_{r}\right)$ is bounded and equicontinuous on $[0, T]$, we can even deduce that $Q^{-1} R$ $\left(W_{r}\right) \subseteq P C([0, T] ; X)$ is equicontinuous on $J_{i}, i=0,1,2, \ldots, p$, where $J_{0}=\left(0, t_{1}\right], J_{1}=\left(t_{1}\right.$, $\left.t_{2}\right], \ldots, J_{p-1}=\left(t_{p-1}, t_{p}\right], J_{p}=\left(t_{p}, T\right]$. Because $Q^{-1}$ is Lipschitz continuous with constant $1 /\left(1-M\left(k+\sum_{i=1}^{p} k_{i}\right)\right)$ for $W \subseteq W_{r}$, we obtain that

$$
\beta_{p c}\left(Q^{-1} R W\right) \leq \beta_{p c}(R W) /\left(1-M\left(k+\sum_{i=1}^{p} k_{i}\right)\right) .
$$

Noting that $R W \subseteq C([0, T] ; X) \subseteq P C([0, T] ; X)$, the following conclusion is obvious:

$$
\beta_{p c}(R W) \leq \beta_{c}(R W) .
$$

Thus,

$$
\beta_{p c}\left(Q^{-1} R W\right) \leq \beta_{c}(R W) /\left(1-M\left(k+\sum_{i=1}^{p} k_{i}\right)\right) .
$$

On the other hand, from Lemma 3.1, for $t \in[0, T]$, we know that

$$
\begin{aligned}
\beta(R W(t)) & \leq 4 M \int_{0}^{t} \beta(f(s, W(s)) d s \\
& \leq 4 M \int_{0}^{t} l(s) \beta(W(s)) d s .
\end{aligned}
$$

From Lemmas 2.4 and 2.5, we have

$$
\beta_{c}(R W) \leq 4 l_{1} M \beta_{p c}(W) .
$$

Consequently,

$$
\beta_{p c}\left(Q^{-1} R W\right) \leq 4 l_{1} M \beta_{p c}(W) /\left(1-M\left(k+\sum_{i=1}^{p} k_{i}\right)\right) .
$$


Since $M\left(4 l_{1}+k+\sum_{i=1}^{p} k_{i}\right)<1$, the mapping $Q^{-1} R$ ia a $\beta_{p c}$-contraction in $W_{r}$. By Lemma 2.2, the operator $Q^{-1} R$ has a fixed point in $W_{r}$, which is just the mild solution of nonlocal impulsive problem (1.1). This completes the proof.

Remark 3.1 In many previous articles, such as $[4,11,14,16,19,22-24]$, the authors obtain the existence results under many different conditions. However, they need the compactness of the semigroup $S(t)$ or nonlocal item $g$, or the Lipschitz continuity of $f$. Here, we make use of the technique of operator transformation and the Hausdorff measure of noncompactness to obtain the existence result, without the compactness condition of nonlocal item $g$ and Lipschitz assumption on $f$. Therefore, our result has wide applications. Furthermore, we also have the following conclusions.

First, we list the following hypotheses:

$\left(H_{A}^{\prime}\right)$ The $C_{0}$ semigroup $S(t)$ is compact;

$\left(H_{f}\right)\left(3^{\prime}\right) f(t, \cdot): X \rightarrow X$ is compact for a.e. $t \in[0, T]$.

$\left(H_{f}\right)\left(3^{\prime \prime}\right) f(t, \cdot): X \rightarrow X$ is Lipschitz continuous, i.e., there exists a constant $k^{\prime}>0$ such that $\|f(t, x)-f(t, y)\| \leq k^{\prime}\|x-y\|$ for a.e. $t \in[0, T], x, y \in X$.

Theorem 3.2 Assume that the conditions $\left(H_{A}^{\prime}\right),\left(H_{f}\right)(1)-(3),\left(H_{I}\right),\left(H_{g}\right)$, and $\left(H_{r}\right)$ are satisfied. Then the nonlocal problem (1.1) has at least one mild solution on [0, T].

Theorem 3.3 Assume that the conditions $\left(H_{A}\right),\left(H_{f}\right)(1)(2)\left(3^{\prime}\right),\left(H_{I}\right),\left(H_{g}\right)$, and $\left(H_{r}\right)$ are satisfied. Then the nonlocal problem (1.1) has at least one mild solution on [0, T].

Moreover, if the condition $\left(H_{f}\right)(3)$ in Theorem 3.1 is replaced by $\left(H_{f}\right)(3 ")$, then we obtain the existence result (Theorem 2.1) of [16].

\section{An example}

As an application of Theorem 3.1, we consider the following semilinear impulsive parabolic equation:

$$
\begin{aligned}
& \frac{\partial}{\partial t} w(t, x)=-A(x, D) w(t, x)+F(t, w(t, x)), \quad t \in[0, T], \quad x \in \Omega, \quad t \neq t_{i} \\
& D^{\alpha} w(t, x)=0, t \in[0, T], \quad x \in \partial \Omega \text { for }|\alpha| \leq m \\
& w(0, x)+\sum_{j=1}^{q} c_{j} w\left(s_{j}, x\right)=u_{0}(x), \quad 0<s_{1}<\cdots<s_{q}<T, x \in \Omega \\
& w\left(t_{i}^{+}, x\right)-w\left(t_{i}^{-}, x\right)=I_{i}\left(w\left(t_{i}, x\right)\right), i=1,2, \ldots, p
\end{aligned}
$$

where $\Omega$ is a bounded domain in $R^{n}(n \geq 1)$ with smooth boundary $\partial \Omega, A(x, D) u=\Sigma_{\mid}$ $\alpha \mid \leq 2 m a_{\alpha}(x) D^{\alpha} u$ is strongly elliptic, $u_{0} \in L^{2}(\Omega), F:[0, T] \times R \rightarrow R$ and $c_{j}$ are given real numbers for $j=1,2, \ldots, q$.

Take $X=L^{2}(\Omega)$ and define the operator $A: D(A) \subseteq X \rightarrow X$ by

$$
\begin{aligned}
& D(A)=H^{2 m}(\Omega) \cap H_{0}^{m}(\Omega), \\
& A u=-A(x, D) u .
\end{aligned}
$$

It is turned out that the operator $A$ generates an equicontinuous $C_{0}$-semigroup on $X$. More details about the fact can be found in the monograph of Pazy [25]. This implies that the semigroup $\{T(t), t \geq 0\}$ generated by $A$ satisfies the condition $\left(H_{A}\right)$.

Now, we assume that: 
(1) $f:[0, T] \mathrm{x} X \rightarrow X$ is defined by

$$
f(t, y)(x)=F(t, y(x)), \quad t \in[0, T], x \in \Omega .
$$

(2) $g: P C([0, T ; X]) \rightarrow X$ is a continuous function defined by

$$
g(u)(x)=u_{0}(x)-\sum_{j=1}^{q} c_{j} u\left(s_{j}\right)(x), 0<s_{1}<\cdots<s_{q}<T, \quad x \in \Omega,
$$

where $u(s)(x)=w(s, x), 0<s<T, x \in \Omega$.

(3) $I_{i}: X \rightarrow X$ is a continuous function for each $i=1,2, \ldots, p$, defined by

$$
I_{i}(\gamma)(x)=I_{i}(\gamma(x)) .
$$

Under these assumptions, the above partial differential system (4.1) can be reformulated as the abstract semilinear impulsive problem (1.1), and conditions $\left(H_{f}\right),\left(H_{g}\right)$, and $\left(H_{r}\right)$ are satisfied with $k=\sum_{j=1}^{q}\left|c_{j}\right|,\|g(0)\|=\left\|u_{0}\right\|$. In addition, if the inequality $M\left(4 l_{1}+\sum_{j=1}^{q}\left|c_{j}\right|+\sum_{i=1}^{p} k_{i}\right)<1$ holds, then due to Theorem 3.1, the problem (4.1) has at least one mild solution $u \in C\left([0, T] ; L^{2}(\Omega)\right)$.

\section{Acknowledgements}

This research was supported by the Natural Science Foundation of China (10971182), the Tianyuan Youth Foundation (11026115), the Natural Science Foundation of Jiangsu Province (BK2010309 and BK2009179), the Natural Science Foundation of Jiangsu Education Committee (10KJB110012 and 09KJB110010), the Yangzhou University Scholarship for Overseas Studies and the Natural Science Foundation of Yangzhou University (2010CXJ003 and 2011CXJ002).

\section{Authors' contributions}

All authors read and approved the final manuscript.

\section{Competing interests}

The authors declare that they have no competing interests.

Received: 7 August 2011 Accepted: 14 February 2012 Published: 14 February 2012

\section{References}

1. Ahmed, NU: Optimal feedback control for impulsive systems on the space of finitely additive measures. Publ Math Debrecen. 70, 371-393 (2007)

2. Benchohra, M, Henderson, J, Ntouyas, S: Impulsive differential equations and inclusions. Hindawi Publ Corp. New York (2006). Contemporary Mathematics and its Applications, vol. 2

3. Cardinali, T, Rubbioni, P: Impulsive semilinear differential inclusions: topological structure of the solution set and solutions on non-compact domains. Nonlinear Anal TMA. 69, 73-84 (2008). doi:10.1016/j.na.2007.05.001

4. Fan, ZB, Li, G: Existence results for semilinear differential equations with nonlocal and impulsive conditions. J Funct Anal. 258, 1709-1727 (2010). doi:10.1016/j.jfa.2009.10.023

5. Liu, JH: Nonlinear impulsive evolution equations. Dynam Contin Discrete Impuls Syst. 6, 77-85 (1999)

6. Rogovchenko, Y: Impulsive evolution systems: main results and new trends. Dynam Cont Discrete Impuls Syst. 3, 57-88 (1997)

7. Byszewski, L, Lakshmikantham, V: Theorem about the existence and uniqueness of a solution of a nonlocal abstract Cauchy problem in a Banach space. Appl Anal. 40,11-19 (1990)

8. Ntouyas, S, Tsamatos, P: Global existence for semilinear evolution equations with nonlocal conditions. J Math Anal Appl 210, 679-687 (1997). doi:10.1006/jmaa.1997.5425

9. Byszewski, L, Akca, H: Existence of solutions of a semilinear functional-differential evolution nonlocal problem. Nonlinear Anal. 34, 65-72 (1998). doi:10.1016/S0362-546X(97)00693-7

10. Benchohra, M, Ntouyas, S: Nonlocal Cauchy problems for neutral functional differential and integrodifferential inclusions in Banach spaces. J Math Anal Appl. 258, 573-590 (2001). doi:10.1006/jmaa.2000.7394

11. Dong, QX, Li, G: Existence of solutions for semilinear differential equations with nonlocal conditions in Banach spaces. Electron J Qual Theory Diff Equ. 47, 1-13 (2009)

12. Obukhovski, V, Zecca, P: Controllability for systems governed by semilinear differential inclusions in a Banach space with a noncompact semigroup. Nonlinear Anal TMA. 70, 3424-3436 (2009). doi:10.1016/..na.2008.05.009

13. Xue, XM: Semilinear nonlocal problems without the assumptions of compactness in Banach spaces. Anal Appl. 8 211-225 (2010). doi:10.1142/S021953051000159X

14. Xue, XM: Nonlocal nonlinear differential equations with a measure of noncompactness in Banach spaces. Nonlinear Anal TMA. 70, 2593-2601 (2009). doi:10.1016/j.na.2008.03.046 
15. Xue, XM: $L^{p}$ theory for semilinear nonlocal problems with measure of noncompactness in separable Banach spaces. $J$ Fixed Point Theory Appl. 5, 129-144 (2009). doi:10.1007/s11784-008-0090-5

16. Liang, J, Liu, JH, Xiao, TJ: Nonlocal impulsive problems for nonlinear differential equations in Banach spaces. Math Comput Modelling. 49, 798-804 (2009). doi:10.1016/j.mcm.2008.05.046

17. Banas, J, Goebel, K: Measure of Noncompactness in Banach Spaces, Lecture Notes in pure and Applied Mathematics, vol. 60. Marcel Dekker, New York (1980)

18. Agarwal, R, Meehan, M, O'Regan, D: Fixed Point Theory and Applications, Cambridge Tracts in Mathematics. Cambridge University Press, Cambridge (2001)

19. Bothe, D: Multivalued perturbations of m-accretive differential inclusions. Israel J Math. 108, 109-138 (1998). doi:10.1007/ BF02783044

20. Cardinali, T, Rubbioni, P: On the existence of mild solutions of semilinear evolution differential inclusions. J Math Anal Appl. 308, 620-635 (2005). doi:10.1016/j.jmaa.2004.11.049

21. Kamenskii, M, Obukhovskii, V, Zecca, P: Condensing Multivalued Maps and Semilinear Differential Inclusions in Banach Spaces, de Gruyter Series in Nonlinear Anal. Appl. 7. Walter de Gruyter, Berlin (2001)

22. Aizicovici, S, Staicu, V: Multivalued evolution equations with nonlocal initial conditions in Banach spaces. Nonlinear Diff Equ Appl. 14, 361-376 (2007). doi:10.1007/s00030-007-5049-5

23. $\mathrm{Zhu}, \mathrm{LP}, \mathrm{Li}$, G: On a nonlocal problem for semilinear differential equations with upper semicontinuous nonlinearities in general Banach spaces. J Math Anal Appl. 341, 660-675 (2008). doi:10.1016/j.jmaa.2007.10.041

24. Zhu, LP, Li, G: Existence results of semilinear differential equations with nonlocal initial conditions in Banach spaces. Nonlinear Anal. 74, 5133-5140 (2011). doi:10.1016/..na.2011.05.007

25. Pazy, A: Semigroups of Linear Operators and Applications to Partial Differential Equations. Springer-Verlag, Berlin (1983)

doi:10.1186/1687-1847-2012-10

Cite this article as: Zhu et al:: Impulsive differential equations with nonlocal conditionsin general Banach spaces. Advances in Difference Equations 2012 2012:10.

\section{Submit your manuscript to a SpringerOpen ${ }^{\circ}$} journal and benefit from:

- Convenient online submission

- Rigorous peer review

- Immediate publication on acceptance

- Open access: articles freely available online

- High visibility within the field

- Retaining the copyright to your article

Submit your next manuscript at $\boldsymbol{s p r i n g e r o p e n . c o m ~}$ 